\title{
THE ROLE OF TUBULAR PROJECTIONS OF PNEUMOCYSTIS CARINII IN PATHOGENICITY OF P. CARINII PNEUMONIA
}

\author{
Tsunezo Shiota, Yoshinaru Shimada, Hiroshi Kurimoto, \\ Hiroshi OIKAWA and NAOKI ARIZONO \\ Received May 20 1994/Accepted 22 July 1994
}

\begin{abstract}
The aim of this research is to clarify the role of tubular projections of Pneumocystis carinii in $P$. carinii pneumonia. Rats, mice and cats with $P$. carinii infections were investigated by electron microscopy and semithin section light-microscopy. We found that the tubular projections adhered closely to the type I alveolar epithelial cells in the same manner as the body of trophozoites attached to the type I epithelium. Numerous tubular projections were observed between the type I epithelium and small clusters of trophozoites, and some of these projections adhered closely to the type I epithelium. In the heavily infected alveoli, many tubular projections were found around the trophozoites. These projections are likely to link the organisms, creating a big mass of organisms too big to be discharged from the lung by the surfactant alveolar clearance mechanism. We think that the tubular projections may play a role in the adherence of $P$. carinii to the type I epithelium as well as in formation of large masses of these organisms that cannot be cleared from alveoli. This can then give rise to an increasingly severe respiratory failure.
\end{abstract}

\section{INTRODUCTION}

The development of Pneumocystis carinii pneumonia is significantly affected by the host defense mechanisms. It is generally considered that impaired cellular immunity is more important than impaired humoral immunity in predisposing to $P$. carinii infection. On the other hand, the parasitic behavior of $P$. carinii is also thought to be critical for the manifestation of its pathogenicity. P. carinii has been reported to be adherent to type I alveolar epithelial cells and form a significantly large mass in the alveoli. This parasitic behavior seems to be related to interference with gas diffusion and failure of ventilation. The pellicle of $P$. carinii trophozoite carries tubular projections, called tubular expansions, that show the same structure as the pellicle from which they arise (Vavra and Kucera, 1970; Ham et al., 1971; Vossen et al., 1978). Freeze-fracture replication studies of $P$. carinii revealed that the projection could be classified morphologically into four types as follows: tubular, lobopodia, branching and beaded structures (Yoshikawa et al., 1987). However, the role of tubular projections in the pathogenicity of $P$. carinii pneumonia is not clear.

In this study, we show that the tubular projections are one of the specialized structures which allow $P$. carinii to fix themselves to type I epithelial cells of alveoli that expand and contract during respiration. Furthermore, the projections around $P$. carinii may also adhere to each other giving rise to a big mass of $P$. carinii that hinders respiratory air exchange.

\section{MATERIALS AND METHODS}

The methods of provoking pulmonary alveolus filling with $P$. carinii in cortisone-treated rats, conventionally raised nude mice and corticosteroid-treated cats have been described (Frenkel et al., 1966; Shiota et al., 1986, 1990). For the electron microscopic inventigations the lungs were removed and cut into small pieces. These tissue fragments were then put in a specially designed plastic syringe containing $2.5 \%$ glutaraldehyde in cacodylate buffer $(0.05 \mathrm{M}, \mathrm{pH} 7.4)$, and air was removed from the alveoli by negative pressure produced by withdrawal of the plunger (Ebe et al., 1968; Takeuchi, 1980). The tissues were immersed in the same fixative for 3 hours at $4^{\circ} \mathrm{C}$ and washed with the same buffer containing $8.2 \%$ sucrose. Then the tissues were dehydrated through a series of acetone and embedded in epoxy resin. Ultrathin sections were cut with a diamond

Department of Medical Zoology, Kyoto Prefectural University of Medicine, Kawaramachi Hirokoji, Kamikyoku, Kyoto 602, Japan 


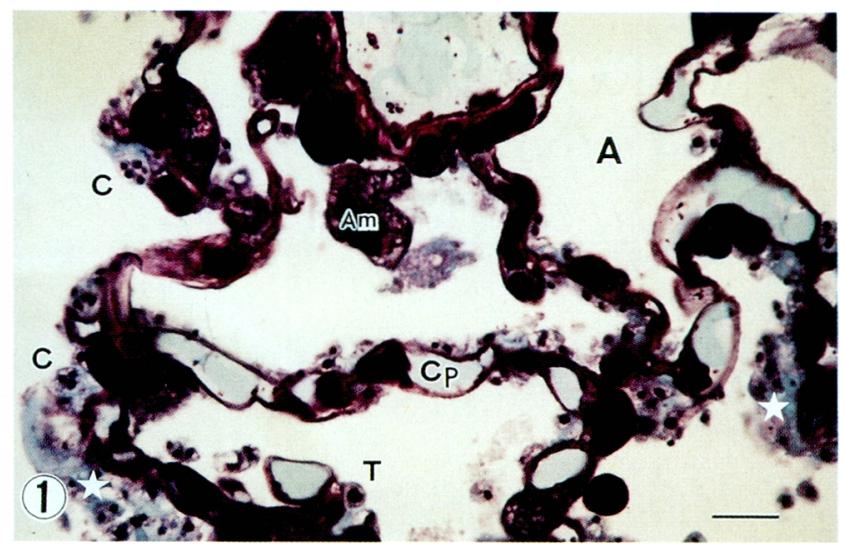

Figure 1 JB-4 plastic-embedded $1 \mu \mathrm{m}$ thick section of lightly infected alveoli from the lung of a rat (after five weeks of corticosteroid treatment). In this figure, about 80 trophozoites $(\mathrm{T})$ and 2 cysts $(\mathrm{C})$ are observed along the surface of epithelial cell. A, air space of alveoli; $\mathrm{Cp}$, capillary; Am, alveolar macrophage; *, a small cluster of $P$. carinii; Scale bar, 10 $\mu \mathrm{m}$. (Giemsa).

knife and placed on uncoated copper drids. The ultrathin sections were stained with uranyl acetate and lead citrate, and observed under a JEOL 100S electron microscope. The procedures for light microscopic investigations of Giemsa stained $1 \mu \mathrm{m}$ sections embedded in JB-4 plastic were briefly as follows. The sections were stained with Giemsa (diluted 20 times with pH 7.2 PBS). More than three hours staining in an incubator at $37^{\circ} \mathrm{C}$ was required to obtain satisfactory results because the sections were so thin (Shiota, 1984).

\section{RESULTS}

Lightly infected alveoli:

Lightly infected alveoli were examined in $1 \mu \mathrm{m}$ section embedded in JB-4 plastic and stained with Giemsa. As shown in Figure 1, the alveolar septa were almost normal, and contained much air. The alveolar capillary membrane (respiratory membrane) and blood capillaries were well preserved. Trophozoites and cysts were found to be closely attached to the type I epithelium. Measurements of the respiratory membrane in Figure 1 showed that about 20\% of the entire membrane was covered with the organisms.

In TEM sections, fine structures of $P$. carinii were similar to those reported previously (Vavra and Kucera, 1970). A membrane to membrane attachment was observed between the trophozoite of $P$. carinii and type I epithelium (Figure 2). A few tubular projections

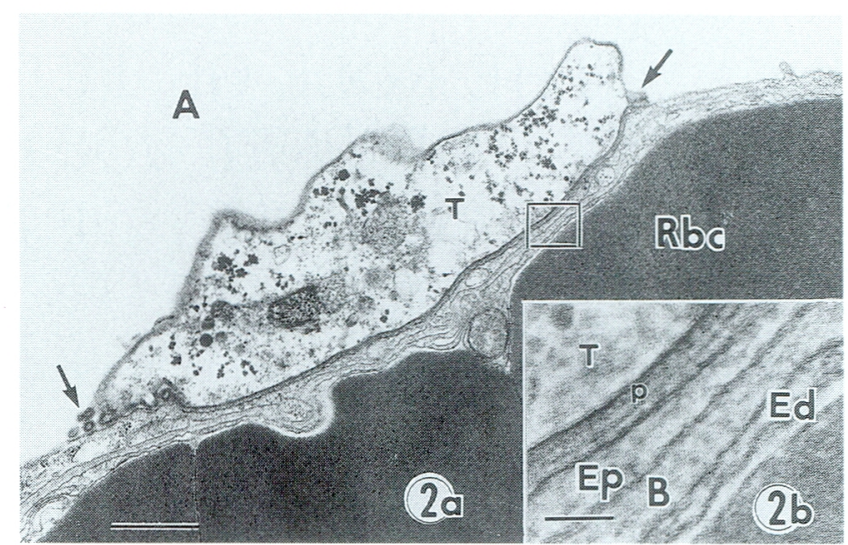

Figure 2a-b Cat, corticosteroid treated for five months. 2a: A few tubular projections (arrows) are found at the peripheral attachment surface of a small trophozoite $(\mathrm{T})$ adhering to the respiratory membrane. 2b: Higher magnigication of the area in the square in Figure 2a shows that the trophozoite is attached to the respiratory membrane. A, air space of alveolus; P, pellicle of the trophozoite; Ep, cytoplasm of type I epithelium; B, basement membrane; Ed, endothelial cell; Rbc, red blood cell. Scale bar, $100 \mathrm{~nm}$.

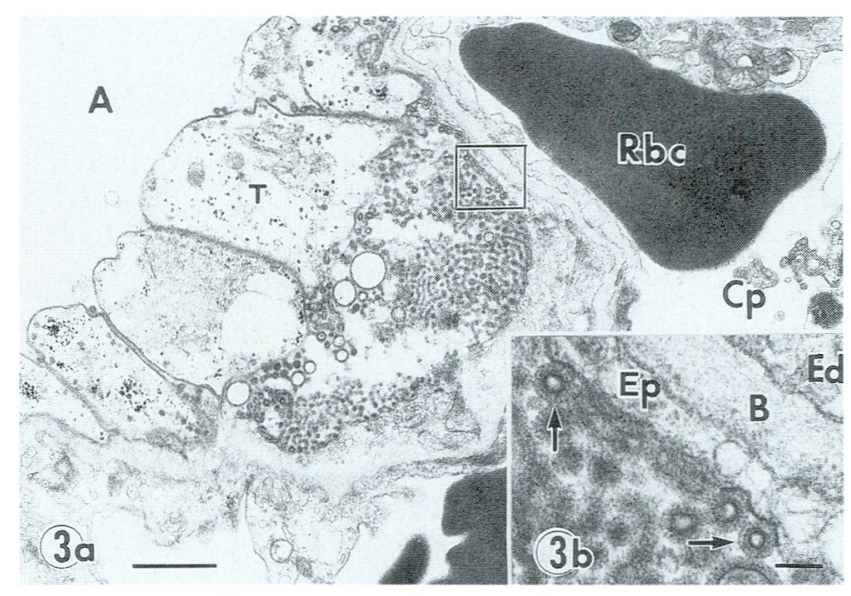

Figure $3 \mathrm{a}-\mathrm{b}$ Nude mouse, five months old, conventionally raised. 3a: Many tubular projections developing between a small cluster of trophozoites $(\mathrm{T})$ and the respiratory membrane. $3 \mathrm{~b}$ : Higher magnification of the area in the square of Figure 3a showing some of the projections (arrows) adhered closely to the type I epithelium. A, air space of alveolus; $\mathrm{Rbc}$, red blood cell; $\mathrm{Cp}$, capillary; Ep, cytoplasm of type I epithelium; B, basement membrane; Ed, endothelial cell. Scale bar, $100 \mathrm{~nm}$.

were found peripheral to the attachment surface of trophozoites (Figure 2). Small cllusters of trophozoites frequently showed much better developed tubular projections than the solitary single trophozoite. Tangles of 


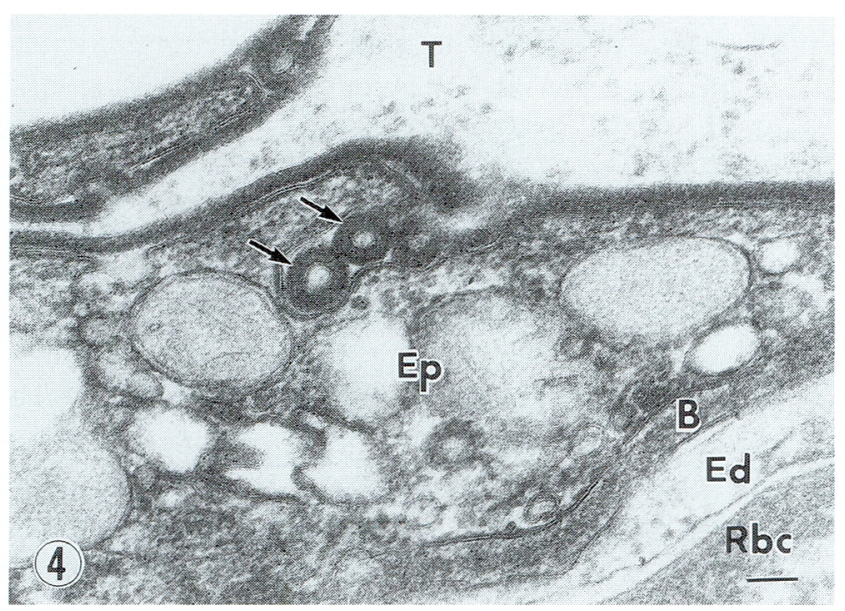

Figure 4 Cat, corticosteroid treated for five months. Some tubular projections (arrows) invaginated in the type I epithelium. T, trophozoite; Ep, cytoplasm of type I epithelium; B, basement membrane; Ed, endothelial cell; Rbc, red blood cell. Scale bar, 100 nm.

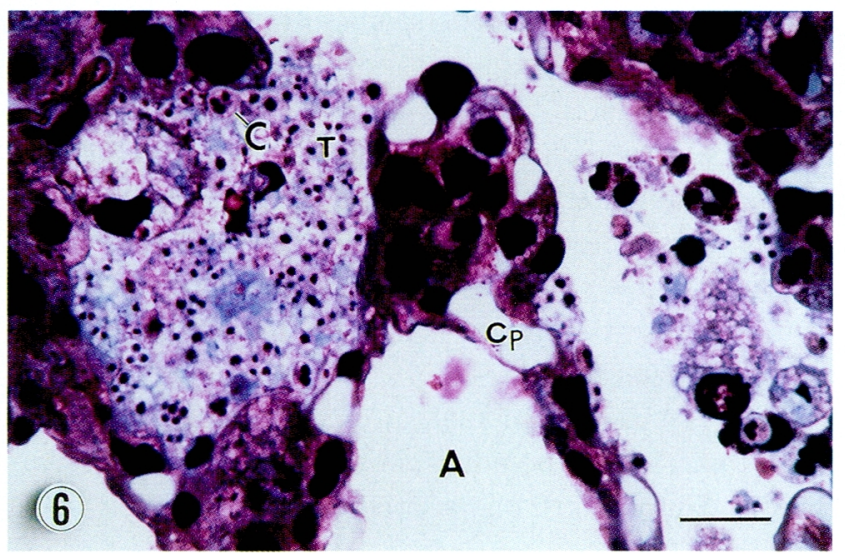

Figure 6 Rat, corticosteroid treated for five weeks. Many trophozoites (T) and cysts (C) are forming a big mass of organisms in heavily infected alveoli. The alveolar air space (A) was almost completely occupied with the clumped trophozoites and cysts. Cp, capillary. Scale bar, $15 \mu \mathrm{m}$. (Giemsa).

numerous projections were observed between the trophozoites and type I epithelium (Figure 3a). Some of the projections adhered closely to the type I epithelium in the same manner as the trophozoites attached to its surface. That is, the surfaces of the organism and the host cells were closely opposed without fusion of the cell membranes (Figure 3b). Some tubular projections invaginated into the type I epithelium so that the epithelial cells became wider (Figure 4). A few tubular projections were also found peripheral to cysts adhering to the type I epithelium (Figure 5). The cyst adhered

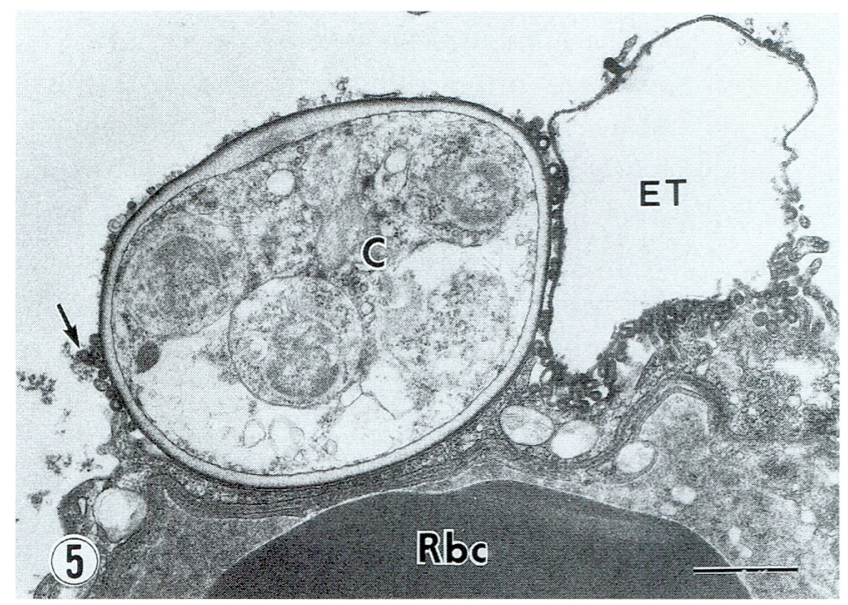

Figure 5 Cat, corticosteroid treated for five months. A few tubular projections (arrow) are found on the side of the cyst (C) adhering to the respiratory membrane. An empty trophozoite (ET) with many tubular projections is seen next to the cyst. Rbc, red blood cell. Scale bar, $1 \mu \mathrm{m}$.

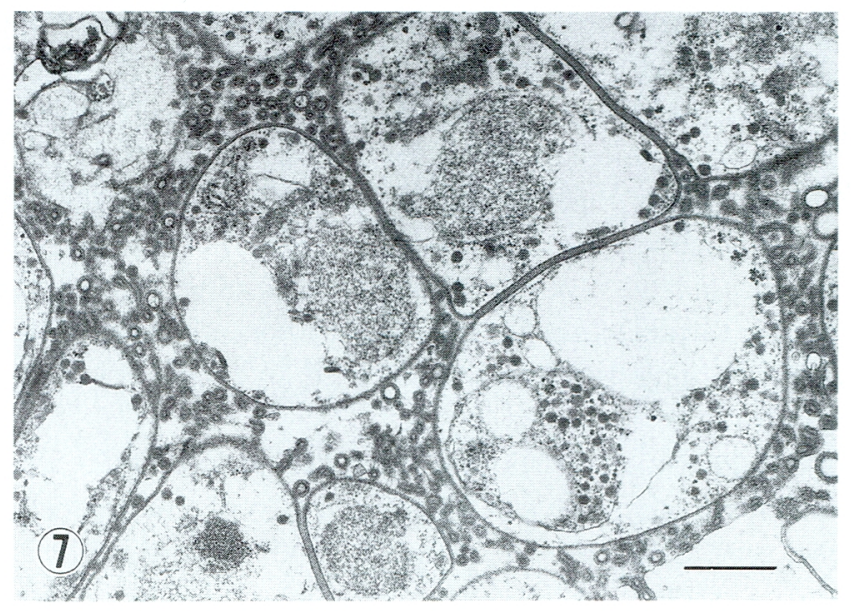

Figure 7 Nude mouse, five months old, conventionally raised. Many tubular projections are found around the trophozoites filling the heavily infected alveolus. Scale bar, $1 \mu \mathrm{m}$.

closely to the type I epithelium in the same manner as the single trophozoite shown in Figure 2a. An empty trophozoite with many tubular projections was often seen beside such cysts (Figure 5).

\section{Heavily infected alveoli:}

In the JB-4 plastic embedded sections, many alveoli were filled with trophozoites, cysts, debris of the host cells and occasionally with foamy macrophage (Figure 6). In TEM sections, many tubular projections were found around the many trophozoites filling the alveolus 
(Figure 7). These tubules showed some variation in diameter and were often filled with dense granular materials. However, they contained no microtubules, intermediate filaments or microfilaments. The tubular projections were not found on the surface of intracystic bodies (daughter cells) in the mature cysts. The structure of tubular projections and the mode of abhesion to the type I epithelium was not different among rats, nude mice and cats.

\section{DISCUSSION}

The pellicle of $P$. carinii trophozoites consists of a 25-30 nm thick layer in which strata can be distinguished: at the inner side a unit membrane as the boundary of the cytoplasm and on the outside a 20-25 $\mathrm{nm}$ thick layer of moderately electron-dense material. This electron-dense outer layer contains a considerable amount of carbohydrates whith bind to Concanavalin A and a lectin from Macura ponrifera (Yoshikawa et al., 1988). These carbohydrates are known to play many roles in cell to cell adhesion. In $P$. carinii cyst walls, glucose, mannose and galactose were found and these sugars may mediate $P$. carinii host interaction (De Stefano et al., 1990). A major surface glycoprotein (gp 120 ), which was of high-mannose type, was detected in $P$. carinii, and a possible role for the adherence of $P$. carinii through this glycoprotein to host mucin components in lung tissue was indicated by Radding et al (1989)

Numerous reports have demonstrated that $P$. car inii attach themselves to a specific alveolar lining cell, the type I epithelium (Barton and Campbell, 1969; Vavra and Kucera, 1970; Ham et al., 1971; Lanken et al., 1980; Yoneda and Walzer, 1980, 1981; Yoshida et al., 1984; Henshaw et al., 1985). P. carinii is tightly attached to the type I epithelium, as judged by freeze -fracture electron microscopy (Yoneda and Walzer, 1983). The interaction of $P$. carinii with this cell plays a central role in the host-parasite relationship in the infection (Walzer, 1986). Tubular projections hava been knows for a long time (Yoneda and Walzer, 1980; Yoshida et al., 1984; Henshaw et al., 1985). P. carinii can alter its morphology to fit the contour of its immediate surroundings. Indeed, the analogy of a rock climber tenaciously grasping the side of a steep incline may well reflect the situation with $P$. carinii (Long et al., 1986). Some authors clearly show these structures, but do not explain the process.

It seemed that $P$. carinii depends on the adherence of the pellicles to the type I epithelium for its par- asitism in the alveoli. The type I epithelium extends and contracts when alveoli expand and contract with breathing. Thus, it seems that the tubular projections may help to fix the organisms to the motile type I epithelium, especially when the number of organisms had increased to fill the alveoli and many organisms had contact with type I epithelium.

In the lightly infected lungs, gas diffusion through the respiratory membrane may be interfered with by the membrane to membrane attachment of $P$. carinii and by limiting the functional area of the respiratory membrane. Furthermore, the large tangles of tubular projections, which were often found between the clusters of trophozoites and respiratory membrane, may also interfere with efficient ventilation locally.

In heavily infected lungs almost all alveoli are filled with trophozoites, cysts, debris of the host cells and occasionally with foamy macrophages (Shiota, 1984). In the present study, we found that many tubular projections developed around the trophozoites and filled the alveoli. These projections seem to link the organisms together, creating a large mass of these organisms in the alveoli anchored to the type I epithelial cells. Because clearance of alveoli normally depends on macrophages which are inhibited by immunosuppression, these masses cannot be cleared. The mixture of organisms and macrophages would need to advance into the bronchioles before becoming subject to removal by ciliary action and coughing.

In conclusion, tubular projections may play an important role in the adhesion of $P$. carinii to the type I epithelium and to one another preventing their being cleared.

\section{ACKNOWLEDGMENTS}

We thank Emeritus Professor Dr. J. K. Frenkel, Dept. of Pathology, Univ. of Kansas Medical Center, Kansas City, Kansas, for his critical reading and comments on the manuscript.

1) Barton, E. G. Jr. and Campbell, W. G. Jr. (1969): Pneumocys tis carinii in lungs of rats treated with cortisone acetate: Ultrastructural observations relating to the life cycle, Amer. J. Pathol., 54, 209-236

2 ) De Stefano, J. A., Cushion, M. T., Puvanesarajah, V. and Walzer P. D. (1990): Analysis of Pneumocystis carinii cyst wall. II. Sugar composition, J. Protozool., 37, 436-441

3 ) Ebe, T., Sakashita, I. and Matsukawa, T. (1968): A new method to fix lung tissues. J. Electron. Microsc., 17, 235-236

4) Frenkel, J. K., Good, J. T. and Shultz, J. A. (1966): Latent pneumocystis infection of rats, relapse, and chemotherapy, 
Lab. Invest., 15, 1559-1577

5 ) Ham, E. K., Greenberg, S. D., Reynolds, R. C. and Singer, D. B. (1971): Ultrastructure of Pneumocystis carinii, Exp. Molec. Pathol., 14, 362-372

6 ) Henshaw, N. G., Carson, J. L. and Collier, A. M. (1985): Ultrastructural observations of Pneumocystis carinii attachment to rat lung, J. Infect. Dis., 151, 181-186

7 ) Lanken, P. N., Minda, M., Pietra, G. G. and Fishman, A. P. (1980): Alveolar response to experimental Pneumocystis carinii pneumonia in the rat, Amer. J. Pathol., 99, 561-588

8 ) Long, E. G., Smith, J. S. and Meier, J. L. (1986): Attachment of Pneumocystis carinii to rat pneumocytes, Lab. Invest., 54, 609-615

9 ) Radding, J. A., Armstrong, M. Y. K., Ullu, E. and Richards, F. F. (1989): Identification and isolation of a major cell surface glycoprotein of Pneumocystis carinii, Infect. Immun., 57,2149 $-2157$

10) Shiota, T. (1984): Morphology and development of Pneumocystis carinii observed by phase-contrast microscopy and semiultrathin section light-microscopy, Jpn. J. Parasitol., $133,433-455$

11) Shiota, T., Yamada, M. and Yoshida, Y. (1986): Morphology, development and behavior of Pneumocystis carinii observed by light- microscopy in nude mice, Zbl. Bakt. Hyg., A262, 230 $-239$

12) Shiota, T., Shimada, Y., Kurimoto, H.and Oikawa, H. (1990): Pneumocystis carinii infection in corticosteroid-treated cats, J. Parasitol., 76, 441-445

13) Takeuchi, S. (1980): Electronmicroscopic observation of Pneumocystis carinii, Jpn. J. Parasitol., 29, 427-453

14) Vavra, J. and Kucera, K. (1970): Pneumocystis carinii Deranoë, its ultrastructure and ultrastructural affinities, J. Protozool., 17, 463-483

15) Vossen, M. E. M. H., Beckers, P. J. A., Meuwissen, J. H. E. T. and Stadhouders, A. M. (1978): Developmental biology of Pneumocystis carinii, an alternative view on the life cycle of the parasite, Ztschr. Parasitenkd., 55, 101-118

16) Walzer, P. D. (1986) Attachment of microbes to host cells: Relevance of Pneumocystis carinii, Lab. Invest., 54, 589-592

17) Yoneda, K. and Walzer, P. D. (1980): Interaction of Pneumocystis carinii with host lungs: an ultrastructural study, Infect. Immun., 29, 692-703

18) Yoneda, K. and Walzer, P. D. (1981): Mechanism of pulmonary alveolar injury in experimental Pneumocystis carinii pneumonia in the rat, Br. J. Exp. Pathol., 62, 339-346

19) Yoneda, K. and Walzer, P. D. (1983): Attachment of Pneumocystis carinii to type I alveolar cells: studied by freeze-fracture electron microscopy, Infect. Immun., 40, 812 $-815$

20) Yoshida, Y., Matsumoto, Y., Yamada, M., Okabayashi, K., Yoshikawa, H. and Sakazawa, M. (1984): Pneumocystis carinii: Electron microscopic investigation on the interaction of trophozoite and alveolar lining cell, Zbl. Bakt. Hyg., A256, 390-399

21) Yoshikawa, H., Morioka, H. and Yoshida, Y. (1987): Freeze -fracture studies on Pneumocystis carinii. II. Fine structure of the trophozoite, Parasitol. Res., 73, 132-139
22) Yoshikawa, H., Morioka, H. and Yoshida, Y. (1988): Ultrastructural detection of carbohydrates in the pellicle of Pneumocystis carinii, Parasitol. Res., 74, 537-543 